\title{
Pragmática de la desinformación. Un estudio del leak de Prism a través de las reacciones de los gobiernos de Estados Unidos, Alemania y Venezuela
}

\section{Desinformazioaren pragmatika. Prism-en leak-en azterketa Estatu Batuetako, Alemaniako eta Venezuelako gobernuen erreakzioen bidez}

\section{Pragmatics of misinformation. A study of the Prism leak through the reactions of the governments of the United States, Germany and Venezuela}

\section{Luis M. Romero Rodríguez ${ }^{1}$ Manuel López Muñoz ${ }^{2}$}

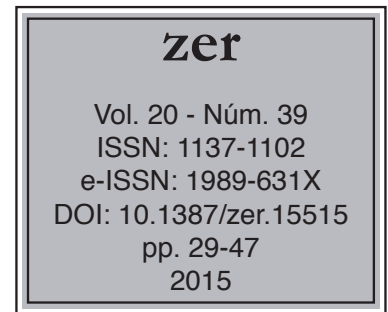

Recibido el 22 de agosto de 2014, aceptado el 6 de mayo de 2015.

\section{Resumen}

En este trabajo se exponen las técnicas de desinformación más comunes que se evidencian en el leak sobre el proyecto Prism de la NSA y sus inmediatas reacciones por parte de los gobiernos de Estados Unidos, Alemania y Venezuela. Para lograr este objetivo, se ha realizado un análisis cualitativo de base interpretativa al discurso de los mandatarios de los tres países, a través de las informaciones recogidas en medios digitales, mediante la localización, criba especializada, métodos de criterio de fuentes y selección aleatoria por metodología de semana construida. Los resultados evidencian el uso de al menos 11 estratagemas de desinformación.

Palabras clave: Desinformación, engaño, manipulación informativa, Prism, Snowden, discurso.

\footnotetext{
Universidad de Huelva, lmiguel.rrodriguez@alu.uhu.es

2 Universidad de Almería, manuel.lopez@ual.es
} 


\title{
Laburpena
}

Lan honetan NSAko Prism proiektuari buruzko leak-en agerian dauden desinformazio-teknika ohikoenak azaltzen dira, bai eta Estatu Batuetako, Alemaniako eta Venezuelako gobernuen berehalako erreakzioak ere. Helburu hori lortzeko, hiru herrialde horietako agintarien diskurtsoaren azterketa kualitatiboa egin da, oinarri interpretatiboa duena, hedabide digitaletan jasotako informazioen bidez. Lokalizazioa, galbahe espezializatua, iturrien irizpidearen metodoak eta eraikitako astearen metodologiaren bidezko ausazko hautaketa egin da. Emaitzek agerian uzten dute, gutxienez, desinformaziorako 11 amarru erabili direla.

Gako-hitzak: Desinformazioa, engainua, manipulazio informatiboa, Prism, Snowden, diskurtsoa.

\begin{abstract}
This paper will present the most common disinformation techniques that are evident in the leak of the NSA Prism project and their immediate reactions from the governments of the United States, Germany and Venezuela. To accomplish this goal has been carried out qualitative interpretive analysis based on the speech of the leaders of the three countries in reference to the information collected through digital media, by locating specialized sieve test methods and sources random selection constructed week methodology. The results show the use of at least 11 stratagems of disinformation.
\end{abstract}

Keywords: Disinformation, deceit, manipulation of information, Prism, Snowden, speech. 


\section{Introducción}

La privacidad de cualquier persona u organización en el mundo es una entelequia y así ha sido demostrado por Edward Snowden, un ex empleado de la consultora de tecnología Bozz Allen Hamilton, que, trabajando bajo contrato en la sede de Hawái de la National Security Agency (NSA), reveló la existencia de un programa denominado Prism que recolecta datos de internet por acceso directo a contenido de correos electrónicos, transferencias de archivos, documentos en la nube y chats de los principales proveedores estadounidenses tales como Google, Facebook, Yahoo, Microsoft, Youtube, Skype, Apple, entre otros.

El 7 de junio de 2013 se da a conocer la información a través de una noticia en la cual se explicaba el "leak confirmado" de 41 diapositivas supuestamente entregadas por Snowden a The Guardian y al Washington Post, cuyo contenido está relacionado con la formación del personal de la agencia en el correcto manejo del programa para "colectar información directamente desde los servidores"3.

Mientras las compañías proveedoras de servicios electrónicos negaban su participación en este programa, los responsables de la inteligencia estadounidense confirmaban la existencia del mismo, justificándolo en razón de la seguridad nacional, en la lucha contra el terrorismo y haciendo énfasis en la aprobación obtenida en el Senado de los Estados Unidos a dichas actuaciones en base a lo contenido en la sección 215 del Patriot Act y en la sección 702 del acto de enmienda del Foreign Intelligence Surveillance Act ${ }^{4}$.

Menor polémica generó la divulgación de la orden judicial secreta de la Foreign Intelligence Surveillance Court, de fecha 12 de abril 2013, en la cual se solicita a la empresa de telefonía Verizon remitir a la NSA "ongoing daily basis" -es decir, de forma regular- las grabaciones de todas las llamadas telefónicas y sus metadatos, tanto de las conversaciones locales dentro de los Estados Unidos, como aquellas internacionales que tuvieran conexión desde y hacia los Estados Unidos, bajo la premisa obligatoria de la confidencialidad de la orden ${ }^{5}$

En el caso anterior, aunque se ha alegado que el gobierno estadounidense no tiene acceso directo a la información obtenida de las llamadas telefónicas, ya que las mismas son depuradas en virtud de términos de búsqueda, analizadas y auditadas por un pequeño número de oficiales entrenados para ese trabajo, con el objeto de identificar posibles atentados terroristas ${ }^{4}$, no es menos cierto que dicha justificación legal podría dar cabida a la legitimación del espionaje internacional, incluyendo información de gobiernos, empresas y personas fuera de la propia jurisdicción territorial de los Estados Unidos.

Esta orden, así como cualquiera que contravenga los derechos de intimidad y privacidad van en contra del propio marco constitucional estadounidense, al es-

\footnotetext{
${ }^{3}$ GREENWALD, G. \& MACASKILL, E. (2013). NSA Prism program taps in to user data of Apple, Google and others. [En línea] Disponible en: http://goo.gl/dJdkD [Fecha de consulta: 6 noviembre 2013].

4 ROBERTS, D. \& SPENCER, A. (2013). US intelligence outlines checks it says validate surveillance. [En línea] Disponible en: http://goo.gl/fuTtv1 [Fecha de consulta: 09 julio 2013].

5 VINSON, R. (2013). Secondary Order: Foreign Intelligence Surveillance Court. [En línea] Disponible en: http://goo.gl/5evzC [Fecha de consulta: 9 julio 2013].
} 
tar en clara contradicción con la cuarta enmienda (incluida en el Bill of Rights de 1791), aunque aplican ciertas condiciones para la excepción al principio constitucional, como las establecidas en la reforma de la Foreign Intelligence Surveillance Act (FISA) de $2007^{6}$, que reduce las restricciones y controles previos a la vigilancia de sospechosos de terrorismo en la cual, al menos uno de ellos se encuentre en espacio extranjero.

Es importante destacar que Estados Unidos es el mayor hub de información del mundo debido a razones históricas, económicas, políticas y sobre todo porque las sedes centrales de los grandes proveedores de comunicaciones están radicados dentro de sus fronteras. Esto hace que el ámbito jurisdiccional de las normas faculte a los organismos de inteligencia, sin mayor limitación, a solicitar información a estas empresas de servicios de telecomunicaciones.

Ya en febrero de 2008, un artículo publicado en Institute of Electrical and Electronics Engineers Journal of Security and Privacy, advertía sobre importantes deficiencias en la arquitectura del sistema de vigilancia que pueden crear graves riesgos de seguridad, incluyendo en estos el peligro que dicho sistema pueda ser utilizado por usuarios no autorizados, malversado por personal interno o abusado por el propio gobierno (Bellovin et al., 2008).

La mayoría de los países, gobiernos y personas están relacionadas directa o indirectamente con los servicios de telecomunicaciones con sede en Estados Unidos, por lo que la aplicación jurisdiccional de las directrices del Protect America Act of 2007, afecta la privacidad y confidencialidad de las comunicaciones y archivos electrónicos, tanto de personas como de instituciones, en cualquier parte del mundo que se utilicen estos servicios.

La información brindada por Snowden sale a la luz en un momento complicado para Estados Unidos y su Política Exterior, tanto por las presiones de sus pares asiáticos, las diversas primaveras que sacudían el polvorín del Medio Oriente -Egipto, Siria, Turquía y los territorios ocupados de Iraq y Afghanistán-, así como por su propia situación económica y social interna, que había dado cabida al surgimiento de movimientos sociales en Washington y Wall Street. Indudablemente el leak sobre Prism había fermentado viejas rencillas exacerbadas que se suponían agotadas después de la caída del muro de Berlín, más aún cuando ya era una costumbre generalizada referirse a Estados Unidos como la Policía del Mundo.

El material hecho público por Snowden abrió un debate en el que la sociedad comenzó a replantearse cuáles eran los límites de sus espacios personales, sobre todo por la profundización de la dicotomía privacidad-seguridad que comenzó a tomar forma el 11 de septiembre de 2001 (Greene, 2014: 795-826; Preibusch, 2015: 48-55 ). La idea de un gran hermano que vigila desde un panóptico sin controles ni límites, parece materializarse fuera de las obras de Orwell, Huxley o Foucault, mientras que gobiernos de distintos países asumían posiciones en contra de las acciones de espionaje de Estados Unidos, algunos alegando desconocimiento, mientras otros cáusticamente las condenaban, aunque en sus respectivos países no se respetasen los derechos invocados de intimidad y privacidad.

${ }^{6}$ Esta ley llevaría por nombre posteriormente "Protect America Act" (2007), cuyo nombre ya da clara evidencia de la necesidad de vincular la conculcación de un derecho por justificaciones patrióticas. 


\section{Marco teórico}

El lenguaje es un constructor e institucionalizador de realidades (Gilovich, 1993: 88111; Searle, 1997: 93-123; Watzlawick, 2003; Berger y Luckmann, 2003: 162-202; Romero-Rodríguez, De Casas y Torres-Toukoumidis, 2015: 31-43), por lo que el discurso, sobre todo aquel mediatizado y proferido por sujetos con autoridad y reconocimiento, crea en la sociedad un entendimiento relativo sobre las situaciones que los rodean. Esto quiere decir que aunque lo construido mediante la acción discursiva sea o no reflejo de una realidad más o menos objetiva, se convierte en facultad del emisor la socialización de esa posición o al menos una matriz informativa imperante, más aun en un ecosistema comunicativo caracterizado por la mediamorfosis donde la ingente cantidad de informaciones a las que estamos expuestos, aunado a la crisis de los medios convencionales y el prosumo digital, hacen que la desinformación sea más una característica propia del sistema que una excepción a la norma (Aguaded y Romero-Rodríguez, 2015: 44-57).

La realidad, asumida como una entidad objetiva, viene desde su origen cargada de gravámenes subjetivos, así como de considerables procesos de recodificación, lo que imposibilita la existencia de una sola realidad y mucho menos una verdad absoluta. Sin embargo, es menester comprender que aunque la desinformación sea un elemento más del complejo proceso informativo, existen un conjunto de técnicas y estratagemas pragmalinguiísticas encaminadas volitivamente al engaño y a la distracción de la formulación de realidades más o menos objetivas por parte de las audiencias (Durandín, 1995: 8; Romero-Rodríguez, 2013: 319-342).

Aunque ofrecer una explicación pormenorizada de cada estratagema de desinformación existente en la presente investigación es imposible en razón de sus límites de extensión, ofrecemos a continuación una enumeración y breve descripción de las mismas que servirán posteriormente para el análisis de cada caso aplicado.

\subsection{Técnicas léxico-semánticas}

- Simplificación conceptual: Consiste en eliminar la complejidad de un asunto reduciéndolo al propio campo semántico que se desea simplificar. La simplificación conceptual no admite matices, por lo que todo se reduce a "bueno o malo", aun cuando la realidad sea compartida (RomeroRodríguez, 2015).

- Eufemismo: Es una figura retórica que atenúa un término desagradable o políticamente incorrecto mediante el uso de otro con acepciones menos negativas. Según Allan y Burridge (1991: 11) "un eufemismo es utilizado como alternativa a una expresión negativa, con el fin de evitar pérdida de prestigio u ofender a las audiencias". Se entiende como una subespecie de metáfora evasiva que busca presentar y construir una realidad positiva a la que realmente es negativa, por lo que es una manipulación del lenguaje con efectos desinformativos. Frases como "intervención militar" -en vez de guerra o invasión-, "daños colaterales" -en sustitución de bajas civiles- van haciéndose cada vez mayor eco en nuestro 
ecosistema informativo, mientras se esconde en el propio lenguaje una realidad mucho más compleja que la reflejada en el análisis semántico del término.

- Efecto placebo: Se definen como "palabras teloneras de abuso y agresión" (Grijelmo, 2000), un procedimiento característico de la justificación de alianzas y de las actividades defensivas de ciertas potencias. La existencia de palabras hace creer en la existencia de sucesos y al repetir dichas palabras se instala en el marco de referencia juicios de existencia y de valor (Durandín, 1995). De esta manera es como el riesgo permanente de terrorismo pudiere funcionar como justificación legítima para cualquier práctica de los servicios de seguridad.

- Adjetivos disuasorios: Son componentes del lenguaje que, cuando en realidad existe la posibilidad de cuestionamientos reflexivos (García Avilés, 2009) no admiten réplica o razonamiento contrario, toda vez que su contundencia eclipsa toda posibilidad de duda. El maximalismo de las aseveraciones componen lexías, unidades léxicas compuestas por monemas con alto índice de inseparabilidad, que forman parte de los discursos y que se adaptan a ellos con la finalidad de persuadir con mayor eficacia a los destinatarios del mensaje. De esta manera, es común encontrar adjetivos como "insoslayable", "inquebrantable", "indiscutible", cuando, en realidad, el tema en cuestión admite y solicita un análisis profundo si se deja de lado la forma del discurso que se le adjudica.

\subsection{Técnicas pragmalingüísticas}

- Refuerzo de la culpabilidad: Presupone que el emisor haga entrever a la audiencia que la culpabilidad de un asunto reside en un tercero, aun cuando en realidad es consecuencia directa de las decisiones del propio emisor (Braiker, 2003). Esta estratagema desinformativa ha sido también el común denominador en casos como el crash financiero de los créditos subprime de Marryl Linch y Lehman Brothers (2007), e incluso de la propia crisis de la burbuja inmobiliaria española (2007-2008), cuestiones en las que la clase política y el establishment descargan la responsabilidad sobre los mercados o al sistema bancario y se declaran no culpables de lo sucedido, aun cuando la responsabilidad es compartida.

- Demonización: Procedimiento atributivo creado en función de intereses individuales o colectivos que consiste en referirse a una persona, institución o ideología con términos peyorativos y disfemismos, al tiempo que se atribuye el emisor a sí mismo la figura de paladín que lucha contra ese "mal" recién inventado (Romero-Rodríguez, 2015: 71-73). El mecanismo de la demonización presupone la identificación de un grupo opuesto al mal y, al colocar al emisor, a la opinión pública y a la opinión publicada en un mismo bando, les transfiere a todos la cualidad de nobleza que, en la mis- 
ma proporción, se le niega al enemigo (García Avilés, 2009). Esta técnica de desinformación es evidente cuando se fija como objetivo estratégico la intervención militar en algún Estado y, para conseguir el consenso de la opinión pública, se diseña una especie de propaganda.

- Mau-Mau: Exacerbamiento de los signos de miedo, violencia e intimidación para aumentar la intensidad de la disuasión (Wolfe, 2011). Esta estrategia de desinformación es explicada por Tom Wolfe a partir del análisis de los Mau-Mau, una organización terrorista keniata de la década de los cincuenta que contribuyó a acelerar la descolonización inglesa de muchos países africanos (incluso Kenia), no tanto por sus actos terroristas sino por la propia leyenda de sus prácticas de canibalismo, torturas y extrema crueldad. En cierta medida, nos recuerda, otra vez, las estrategias que desarrolla Orwell en su 1984, cuando presenta a Emmanuel Goldstein y sus seguidores como fautores de algo que, realmente, no ocurre ni se puede demostrar que haya ocurrido.

- Sobresaturación de información: Consiste en la difusión masiva de noticias falsas o sin contrastar, con la intención de crear un estado determinado en la opinión pública. Generalmente esta estratagema se utiliza con el fin de ocultar otras informaciones que pueden ser perjudiciales para el emisor, por lo que intenta introducir en la matriz de opinión estas noticias para que se mantengan más en el ecosistema comunicativo, mientras que aquellas que van en contra de los intereses pasan desapercibidas o tienen menos cubertura (García Avilés, 2009).

- Realidades Inventadas. Las palabras crean las realidades que ellas mismas describen, por lo que su uso común comienza a encerrar otras realidades inherentes a su propia definición, haciendo imperceptible e inapreciable el contenido de la misma. Esta situación coadyuva a que el proceso de significación resulte difuso en la interpretación de la realidad (Searle, 1997). De esta manera, el futuro y no el pasado es lo que determina los efectos en el presente por lo que un acto es el resultado de una profecía que se autocumple, mas no es ni verdadero ni falso sino simplemente creado (Gilovich, 1993: 88-111; Searle, 1997: 93-123; Watzlawick, 2003; Berger y Luckmann, 2003: 162-202).

- Omisión: Se fundamenta en la ocultación total o parcial de información (García Avilés, 2009), de manera que la comprensión de los acontecimientos y su contexto sean ininteligibles ante los intentos por su análisis.

- Distracción: Es un elemento primordial de control social que consiste en desviar la atención de los problemas que afectan directamente a la opinión pública, creando matrices de opinión con el fin de desalinear la agenda setting a que se avoquen al tema introducido, perdiendo seguimiento y fuerza aquellos sucesos que verdaderamente afectan a la 
sociedad por consecuencia del aluvión informativo y opinativo (Romero, 2015: 53-69).

\section{Materiales y método}

La presente investigación busca examinar cualitativamente, a través del análisis de discurso mediático, las declaraciones emitidas por los máximos representantes gubernamentales de Estados Unidos, Alemania y Venezuela, posterior al conocimiento del leak del proyecto Prism, con el objeto de evidenciar el uso de estratagemas de desinformación. Para alcanzar dicho fin se realizará el análisis de contenido de base interpretativa, entendiendo este tipo de método como que "involve interpretation of findings across studies to generate new inductive understandings of the phenomena, events or experiences" (Saini y Shlonsky, 2012: 31). El análisis de discurso mediático se enfoca en los objetos escondidos que dan forma a la producción y diseminación de las informaciones, pero que no son perceptibles a simple vista, como el estudio del entorno informativo, los hechos colaterales y otros backchannels que se sostienen en la comunicación sobre élites semiformales (Gillespie, 2010; Hindman, 2013; Turow, 2011; Karpf et al., 2015: 1901).

El interés de analizar estas declaraciones y posiciones oficiales surge por la evidente demanda de la sociedad en conocer el grado de penetración que tiene el fenómeno de la desinformación en nuestro acontecer diario, más aun cuando pudieren existir suficientes evidencias de un manejo desproporcional de manipulación informativa en torno a todas las reacciones gubernamentales objeto de estudio. De esta manera, se parte de la hipótesis que existirán suficientes evidencias de la utilización de estratagemas de desinformación en las declaraciones de los mandatarios de los países citados ut supra.

Los sujetos de análisis están conformados por la selección de tres sujetos que tuvieron una activa participación mediática después de conocerse la actividad del programa Prism: a) Barack Obama, Presidente de los Estados Unidos, en función de defensa del programa y como representante del país que ejecutó las acciones de espionaje; b) la Canciller alemana Ángela Merkel, quien primeramente rechazó frontalmente la intervención norteamericana en asuntos extranjeros y posteriormente la justificó en función del descubrimiento de que su gobierno había estado participando activamente en el programa y en último lugar, c) Nicolás Maduro, Presidente de Venezuela, país el cual ofreció asilo a Edward Snowden y criticó duramente la posición de los Estados Unidos, liderando además al «bloque bolivariano» conjuntamente con Ecuador, Nicaragua y Bolivia, para el otorgamiento de asilo. Estos tres sujetos seleccionados nos darán una perspectiva desde las tres posiciones del juego político.

\subsection{Estrategias metodológicas}

El análisis de discurso mediático de las informaciones expuestas en medios digitales será la piedra angular epistemológica del presente estudio, con la pertinencia, suficiencia y objetividad de la fuente como criterios prioritarios de la selección de material (Karpf et al., 2015: 1899-1902). El trabajo será de deducción e inferencia apoyada en la localización, catalogación y revisión documental a través de un 
paradigma explicativo-cualitativo que busca ejercer una labor de aproximación teórica de un fenómeno semiótico, comunicativo y social.

Para la obtención del corpus y la recogida de datos, se procedió a realizar una criba especializada a través del motor de búsqueda Google $\AA$, utilizando los descriptores: a) Prism+Snowden+Barack+Obama; b) Prism+Snowden+Angela+Merkel; y c) Prism+Snowden+Nicolás+Maduro. Asimismo, a través de herramientas de búsqueda, fue limitada la exploración a noticias de medios digitales en el período comprendido entre el 7 de junio y el 7 de agosto de 2013 (2 meses naturales), lo que nos arrojó un total de 1269 informaciones, de las cuales 535 corresponden a Barack Obama, 624 a Ángela Merkel y 137 a Nicolás Maduro. Al resultar la criba tan extensa y cuantitativamente disímil, fueron seleccionadas solo aquellas que se ajustaron a la suficiencia y pertinencia de la fuente informativa en los idiomas inglés y español, así como acotado únicamente a géneros informativos (noticias, entrevistas, reportajes y crónicas). También se procedió a eliminar de la selección aquellas unidades informativas duplicadas, reduciendo por razones de equidad la selección a 100 unidades muestrales aleatorias por sujeto de análisis, lo que representaría 18,69\% de las informaciones totales relativas a Barack Obama, 16,02\% de la criba total resultante de la búsqueda de Ángela Merkel y 72,99\% de los resultados de búsqueda de Nicolás Maduro.

Para el muestreo aleatorio se utilizó la metodología de semana construida (Stempel, 1989), dividiéndose los resultados obtenidos entre las 8 semanas del análisis para evitar la duplicidad informativa así como la concentración noticiosa en un intervalo de fechas específico. En relación a la recogida de datos se utilizó el programa informático MAXQDA ${ }^{\circledR}$ para facilitar tomar notas de campo y editar los textos discursivos. Aunque la estrategia metodológica no contempla la codificación emergente o la clasificación de familias de códigos para alcanzar los resultados del eje de estudio, esta interfaz especializada permitió almacenar ordenadamente los datos, así como buscar y recuperar los fragmentos relevantes del texto, con el fin de clasificarlos en las siguientes unidades hermenéuticas que coinciden con las estratagemas de desinformación explicadas en el marco conceptual de este trabajo: (1) Distracción, (2) omisión, (3) demonización, (4) sobresaturación, (5) pseudo-acontecimiento, (6) realidades inventadas, (7) adjetivos disuasorios, (8) timing, (9) eufemismos, (10) Mau-Mau, (11) simplificación conceptual, (12) reforzamiento de la autoculpabilidad, (13) efecto placebo, (14) sin clasificar.

Los resultados obtenidos a través del análisis, serán expuestos en función narrativa y cronológica (Richardson y Adams-St.Pierre, 2000; Ellis y Bochner, 2006; Lee, 2006; Moral-Santaella, 2015), es decir, mediante la explicación pormenorizada de los acontecimientos más importantes que ocurrieron con cada sujeto muestral en el intervalo identificado meridianamente con anterioridad. Asimismo se expondrán las estratagemas evidenciadas en cada uno de los casos desde una posición crítica y reflexiva.

\section{Resultados}

\subsection{La ofensa-defensiva de los Estados Unidos}

El 7 de junio de 2013, un día después de la filtración de los documentos del programa Prism por el periódico británico The Guardian, el presidente Barack Obama 
argumenta que el espionaje de las telecomunicaciones es legal, pues ha sido aprobado por el Congreso y las cortes. Algunos medios de comunicación se hicieron eco en los días posteriores a estas declaraciones intentando minimizar el impacto, pues explicaron que lo contenido en la Protect America Act of 2007 es, como todo acto legislativo, materia de dominio público, por lo que los servicios diplomáticos y consulares de cada Estado acreditados en Washington debían conocer que los servicios secretos de los Estados Unidos tienen acceso a las plataformas informáticas y redes sociales, así como las telecomunicaciones intermediadas por proveedores estadounidenses. Asimismo se evidenciaron en el análisis de las unidades informativas sucintas explicaciones sobre los contratos y acuerdos de los proveedores de servicios de telecomunicaciones y plataformas informáticas, en los que el usuario acepta que el proveedor pueda recabar datos de sus cuentas personales, por lo que narrativamente el espionaje no era tal, pues contaba con la voluntad de los usuarios.

La línea informativa de la Casa Blanca entre el 7 y el 16 de junio, estuvo definida en función de dos declaraciones concedidas por Obama:

(1) "What you've got is two programs that were originally authorized by Congress, have been repeatedly authorized by Congress (...) There are a whole range of safeguards involved. And federal judges are overseeing the entire program throughout"?.

(2) "You can't have 100 percent security and then also have 100 percent privacy and zero inconvenience. You know, we're going to have to make some choices as a society"7.

De esta manera, se evidencia que el gobierno de los Estados Unidos ha adoptado la posición de víctima, ha redefinido el espionaje como actividad esencial para garantizar su homeland security, la protección frente a sus enemigos internos y externos, y ha dejado entrever que, lejos de ser ética o legalmente cuestionable, las actuaciones de sus servicios de inteligencia constituyen la primera y última línea de defensa ante las amenazas constantes a la seguridad y al propio American way of life.

Entre el 9 de junio y el 11 de julio, los debates legislativos en el Congreso y el Senado aumentaban la tensión entre los representantes del partido demócrata (gobierno) y republicano (oposición) por las formas, más que por el fondo, pues ambas administraciones eran corresponsables de la aplicación del espionaje. De los códigos emergentes de las unidades informativas sobre los debates en el Congreso, la mayoría encontró una justificación del espionaje bajo la simplificación conceptual dicotómica: privacidad vs. seguridad.

Aun cuando la aparición de Snowden en el panorama discursivo social presenta una realidad que va mucho más allá de un informante, verbigracia, recordar que ninguna comunicación en el mundo es hermética, se intenta reducirlo en el ecosistema

BAKER, P. \& SANGER, D. (2013). Obama Calls Surveillance Programs Legal and Limited. [En línea] Disponible en: http://goo.gl/x7X0KY [Fecha de consulta: 9 noviembre 2013]. 
comunicativo de la opinión pública a si la actuación de Snowden fue buena o mala, lo que cierra la puerta a un debate de principios, tanto en los medios de comunicación como a la propia investigación académica. Esta simplificación conceptual discursiva reduce los posicionamientos sociales ergo niegan el análisis de cualquier otra categoría de discusión.

El 13 de junio, el director del FBI, Robert Mueller, informa en el Congreso que se ha abierto una investigación penal contra Snowden por "causar un daño significativo a los Estados Unidos y poner en riesgo la vida de ciudadanos norteamericanos" . De esta manera, se comienza el proceso de solicitud de extradición del filtrador errante, quien se encontraba para los momentos en Hong Kong. Los medios norteamericanos, sobre todo en géneros opinativos incrustados en unidades informativas (esencialmente noticias), incidieron en que a Snowden debía vérsele como un prófugo de la justicia que ponía en riesgo la seguridad de los estadounidenses. La criminalización inmediata de las acciones de Snowden y la falta de un fallo judicial sobre su conducta lo convierten en un fugitivo, quedando meridianamente clara la intención del gobierno de demonizarlo ante la opinión pública nacional e internacional, aparte de medida de presión y coacción disuasoria para evitar que otros países le concedieran asilo político, mientras que es pensable que se tratara también de un aviso bajo el método de Mau-Mau, para evitar futuros replicadores (copycats).

Un caso llamativo ocurre el 18 de junio, cuando el director de la NSA, Keith Alexander, declara en una comisión ad hoc del Congreso que la "vigilancia de las comunicaciones" ha ayudado a prevenir más de 50 potenciales ataques terroristas ${ }^{9}$. Es importante identificar este hito, ya que a partir de estas declaraciones el eufemismo vigilancia de las comunicaciones es tomado como parte del lenguaje oficial. Esta posición retórica minimiza como formulación anestésica el significado de espionaje, mientras lo convierte en un término de valoración positiva (vigilancia).

La semana del 23 de junio estuvo centrada informativamente en la huida de Snowden a Rusia, mientras que Ecuador recibía una solicitud de asilo. El portavoz presidencial, Jay Carney, amenazó a China y a Rusia, prometiendo que sus acciones tendrán un "impacto negativo incuestionable". En estas declaraciones también se comprueba el uso de eufemismos y de la estratagema de Mau-Mau, ya que se trata de una amenaza sin fundamentación en el Derecho Internacional, pero que es formulada con palabras anestésicas.

El editorial del Washington Post del 25 de junio dejaba clara la línea de uno de los mayores emporios comunicacionales de Estados Unidos. El diario advertía al presidente ecuatoriano Rafael Correa que de otorgarle el asilo al ex trabajador de la NSA, reemplazaría al finado presidente de Venezuela, Hugo Chávez, como el "principal demagogo antiestadounidense del hemisferio". Asimismo, entre el 9 y el 13 de julio, la Casa Blanca advierte a los gobiernos de Ecuador, Nicaragua, Bolivia y Venezuela, que no deben otorgarle asilo a Snowden, so pena de "graves sanciones". La creación de hechos noticiosos colaterales tales como la prohibición de sobrevolar algunos países del espacio europeo que se le impuso al vuelo que hacía el presidente boliviano

\footnotetext{
8 ADAMS, P (2013). Snowden leaks caused US 'significant harm' - Mueller. [En línea] Disponible en: http://goo.gl/5NVjnl [Fecha de consulta: 13 noviembre 2013].

9 GERSTEIN, J. (2013). NSA: PRISM stopped NYSE attack. [En línea] Disponible en: http://goo.gl/ YXFDCr [Fecha de consulta: 11 noviembre 2013].
} 
Evo Morales desde Moscú rumbo a La Paz el 9 de julio, obligándolo a efectuar una "escala técnica" de 19 horas en Viena (Austria) y posteriormente en el aeropuerto de las Palmas de Gran Canaria (España), no sólo evidencia la presión diplomática que ejerce Estados Unidos y sus aliados contra los países del bloque bolivariano que tendrían intenciones de otorgar asilo al informante, sino un claro indicio de distracción, haciendo que la atención de los medios siga un camino distinto a la propia discusión sobre la legitimidad del espionaje.

\subsection{The show must go on: El sobre-histrionismo alemán}

Tras la información que Edward Snowden proporcionó el 7 de junio de 2013 a los diarios The Economist y The Washington Post, distintos gobiernos del mundo mostraron su inmediato rechazo e indignación por los actos de espionaje que ha llevado a cabo Estados Unidos a través de los proveedores de telecomunicaciones.

Tal es el caso del gobierno alemán, que el mismo día en que la información se hizo pública, condenó con dureza las actuaciones de la National Security Agency (NSA) y advirtió de las repercusiones de esta conducta en las relaciones comerciales de la Unión Europea y los Estados Unidos ${ }^{10}$. La canciller alemana recalcó que la actuación norteamericana "excede todo lo inimaginable" comparando incluso las acciones de espionaje contra embajadas y gobiernos de la UE como "prácticas propias de la Guerra Fría"11.

Vistas las reacciones de rechazo por parte del gobierno alemán y de su canciller Angela Merkel, Snowden no dudó en hacer ver que Alemania y otros países europeos colaboran activamente y "sin hacer preguntas" con las actuaciones de la NSA a través del intercambio de información. Quedaba claro que las intervenciones se hacían de tal forma que, en el caso de que el programa saliera a la luz, los altos cargos políticos podían alegar su desconocimiento total ${ }^{12}$

Para el 10 de julio de 2013, la Canciller alemana reconoce que: "durante décadas los servicios de inteligencia (alemanes y estadounidenses) han trabajado juntos", alegando en la confesión que el intercambio de información se hace bajo ciertas condiciones reguladas ${ }^{13}$. Continúa alegando Merkel que los Estados Unidos han sido los más fieles aliados durante décadas y justifica las actuaciones de ambas agencias de inteligencia ya que "un país sin el trabajo de los servicios secretos sería demasiado vulnerable".

La intencionalidad de desinformar a la Opinión Pública es clara: la Canciller alemana no sólo debe de conocer los contenidos del Protect America Act of 2007 -tanto como acto legislativo público, sino como acción de un aliado de la OTAN-, sino que se le vincula directamente como cómplice en la propia actividad de espionaje después de haber formulado una enérgica condena de las actuaciones de la NSA. Esto hizo que acabara justificando posteriormente dicha colaboración por recurso a la

\footnotetext{
${ }^{10}$ SÁNCHEZ, R. (2013). Merkel apunta a que Washington hace espionaje industrial. [En línea] Disponible en: http://goo.gl/n70B1g [Fecha de consulta: 10 julio 2013].

${ }^{11}$ EFE/AFP. (2013). La UE pide explicaciones 'inmediatas' a EEUU sobre el presunto espionaje. [En línea] Disponible en: http://goo.gl/0em1H [Fecha de consulta: 15 julio 2013].

12 DER SPIEGEL. (2013) New NSA Revelations: Inside Snowden's Germany File. [En línea] Disponible en: http://goo.gl/GDUCmr [Fecha de consulta: 10 julio 2013].

${ }^{13}$ REUTERS/EP. (2013). Merkel defiende la colaboración alemana con EEUU en asuntos de inteligencia. [En línea] Disponible en: http://goo.gl/IzF48 [Fecha de consulta: 11 julio 2013].
} 
regulación de esa actividad y dándole mayor importancia a la seguridad que brindan los servicios secretos que a los derechos a la intimidad y a la privacidad. Observemos entonces el uso de las siguientes estrategias de desinformación:

Encontramos el recurso al eufemismo en la reacción de la Canciller alemana cuando justifica que el "intercambio de información" -que supone una bidireccionalidad del mismo- se hace bajo "condiciones reguladas". En ambos casos, se elaboran simplificaciones conceptuales con el objetivo de evitar que aparezca en la mente del receptor del mensaje la pregunta sobre la activa colaboración del gobierno germano en el espionaje internacional.

En el caso revisado, la canciller alemana recurre al efecto placebo para enterrar las acusaciones de espionaje contra personas e instituciones públicas y privadas en todo el mundo bajo la premisa de que "un país sin el trabajo de los servicios secretos sería demasiado vulnerable"13. De esta manera, la actividad de espionaje internacional, lejos de generar debates éticos y morales con respecto a la privacidad de las informaciones, busca legitimarse por medio del concepto de seguridad nacional, tanto de los Estados Unidos como de Alemania y sus países aliados.

Las primeras reacciones y condenas de Merkel dejan clara su intención de proceder mediante la técnica de ocultación -omisión-y, así, velar la activa participación que han tenido los servicios secretos germanos con sus pares estadounidenses. El problema del uso de esa técnica es que, al darse a conocer posteriores declaraciones de Edward Snowden sobre la participación activa de la Canciller alemana germana, ésta hubo de aceptar, al menos parcialmente, que así había sido.

Barack Obama, por su parte, apoya a Merkel al reconocer que la canciller "se toma en serio las preocupaciones de sus socios y aliados europeos" $14 \mathrm{y}$, en una reunión llevada a cabo a principios de julio de 2013, ambos mandatarios reiteraban "la importancia de seguir con la estrecha cooperación entre los servicios de Inteligencia en la lucha contra el terrorismo y otras amenazas a la seguridad de Estados Unidos, Alemania y sus aliados" 14 y expresaron su interés conjunto en iniciar un diálogo entre Estados Unidos y la Unión Europea (UE) sobre la recolección y la supervisión de Inteligencia y de cuestiones relacionadas con la privacidad y la protección de datos ${ }^{14}$.

El 19 de julio de 2013, salía a la luz la información de que las tropas de la OTAN apostadas en Afganistán utilizaron los datos del programa Prism e incluso solicitaron a través de su interfaz el control de llamadas telefónicas y correos electrónicos, develaciones que van ocurriendo a cuenta gotas pero que dan cuenta del conocimiento previo que debía tener la máxima autoridad del país sobre la amplitud y ejecución del espionaje internacional estadounidense. El Partido Social Demócrata alemán (SPD) y Los Verdes, ambos en la oposición alemana, exigían claras explicaciones a Merkel, mientras las encuestas de opinión pública daban una gran pérdida de popularidad al gobierno germano. Según un sondeo de la cadena de televisión oficial alemana (ARD), dos terceras partes de los alemanes se encontraban descontentos con la reacción de la canciller. Esta situación genera que el 1 de agosto, Alemania anunciara el rompimiento del acuerdo de cooperación para permitir a los Estados Unidos el acceso a la información recabada por los servicios secretos en su territorio ${ }^{15}$.

\footnotetext{
${ }^{14}$ EUROPAPRESS. (2013). Obama y Merkel acuerdan celebrar una reunión para abordar el espionaje de EEUU. [En línea] Disponible en: http://goo.gl/0M54fG [Fecha de consulta: 10 noviembre 2013].

${ }^{15}$ AFP/DPA. (2013). Alemania rompe un acuerdo de espionaje de la Guerra Fría con EEUU y Reino
} 


\subsection{Wag The Dog en Venezuela}

El 6 de julio de 2013, el Presidente de Venezuela, Nicolás Maduro Moros, le ofrece asilo humanitario a Edward Snowden ${ }^{16}$, cuestión que también harán sus compañeros del bloque del ALBA (Alianza Bolivariana para las Américas), Bolivia, Nicaragua y Ecuador ${ }^{17}$. El hecho ocurre en un entorno de una Venezuela en la que los debates políticos más intensos se llevan a través del espionaje y contraespionaje de partidos políticos, líderes de oposición y personajes relevantes del gobierno. Tal fue el caso de una grabación de 64 minutos reproducida por Ismael García, Diputado opositor, donde se oye a Mario Silva, un polémico expresentador de un espacio televisivo gubernamental y dirigente político oficialista, conversar con un funcionario del servicio de inteligencia cubano sobre conflictos internos en las filas oficiales y enumerar denuncias de casos de corrupción, tráfico de influencias, posesión ilícita de armas de fuego, blanqueamiento de capitales y fondos públicos. La aparición de ese testimonio le asestó un duro golpe a la credibilidad de Mario Silva y a la del partido de gobierno.

Ya antes había actuado el chavismo tras la difusión de grabaciones de dirigentes de la oposición, como ocurrió en los casos de María Corina Machado ${ }^{18}$ y de Henrique Capriles García, padre del líder de la oposición, Henrique Capriles Radonski ${ }^{19}$. Podemos decir, pues, que la cuestión del espionaje de las telecomunicaciones en Venezuela es anterior al surgimiento noticioso del caso Snowden.

El ofrecimiento de asilo político coincide con un momento en el que Venezuela sufre un índice de inflación cercano al $4 \%$ mensual $^{20}$, un estimado de $25 \%$ de desabastecimiento de rubros de uso básico -como papel higiénico-, productos lácteos y cárnicos, un aproximado de 20 mil homicidios anuales, un salario mínimo menor a US\$ 80 mensuales, rumores de corrupción administrativa desatada, racionamiento de la energía eléctrica y continuos debates acerca de la legitimidad de las elecciones presidenciales en las cuales resultó electo el actual mandatario venezolano ${ }^{21}$.

Mientras Nicolás Maduro y el partido oficial venezolano aplaudían la actuación de Snowden para desenmascarar la trama de espionaje del programa Prism, en la Asamblea Nacional venezolana, máximo ente del Poder Legislativo e integrado mayormente por congresistas del partido de gobierno, era presentado el Proyecto de Ley de Infogobierno, que incluye en su artículo 94 lo siguiente:

Unido. [En línea] Disponible en: http://goo.gl/KHRrP9 [Fecha de consulta: 11 noviembre 2013].

${ }^{16}$ EFE. (2013). Maduro ofrece asilo a Snowden para protegerle de la 'persecución de EEUU'. [En línea] Disponible en: http://goo.gl/vz6m5m [Fecha de consulta: 23 julio 2013].

${ }^{17}$ EL PAÍS. (2013). Tres países de la alianza bolivariana ofrecen asilo a Snowden si lo pide. [En línea] Disponible en: http://goo.gl/3fp1P [Fecha de consulta: 23 julio 2013].

${ }^{18}$ EL UNIVERSAL. (2013). Comisión de la AN evaluará audio con conversación de María Corina Machado. [En línea] Disponible en: http://goo.gl/C9eJwO [Fecha de consulta: 23 julio 2013].

19 AVN. (2012). Empresario ofrece "contribución" a padre del candidato Capriles para su campaña. [En línea] Disponible en: http://goo.gl/mA6rbL [Fecha de consulta: 23 julio 2013].

20 AFP. (2013). Inflación de los primeros 4 meses de 2013 se ubica en 12,5\%, según el BCV. [En línea] Disponible en: http://goo.gl/rHHRjf [Fecha de consulta: 23 julio 2013].

${ }^{21}$ EL UNIVERSAL. (2013). Capriles: Maduro claudicará debido a la ilegitimidad y la crisis económica. [En línea] Disponible en: http://goo.gl/7hHoQ [Fecha de consulta: 23 julio 2013]. 
Todo aquel que haya obtenido indebidamente, mediante el uso de Tecnologías de Información, datos o informes que guarden relación con la seguridad y defensa de la Nación, será penado con prisión de diez (10) a quince (15) años. Si los datos o la información obtenida conforme al encabezamiento de este artículo, es divulgada o suministrada a cualquier particular, por cualquier vía, comprometiendo la seguridad y defensa de la Nación, será penado con prisión de quince (15) a veinte (20) años.

Fuera de las propias contradicciones e inconsistencias entre discurso y actuación del gobierno venezolano con respecto al espionaje, que podría asegurar doble moral, podemos también identificar el uso consciente de estrategias de desinformación, empezando por la de distracción. En el caso en análisis, la decisión de otorgar asilo humanitario a Edward Snowden, además de ocasionar el efecto deseado en el establishment venezolano, dejó fuera de las primeras páginas al menos por una semana los temas de la inflación, el desabastecimiento, la delincuencia y la corrupción administrativa, de lo que puede colegirse que se desvió la atención primordial de los temas que verdaderamente afectan el acontecer diario venezolano, al menos en el propio seno del ecosistema comunicativo y de los propios debates en su sociedad.

Aun cuando la sobresaturación de los espacios informativos vienen de la mano de la propia naturaleza del ecosistema actual, hipermediatizado con las redes sociales y la propia inmediatez de la noticia, podemos decir que existe cierta intencionalidad de generar intoxicación informativa con la decisión de otorgar asilo a Snowden por parte del gobierno venezolano, que creó un aluvión de informaciones colaterales, sobre todo géneros de opinión, que daban respuesta a la decisión del gobierno venezolano de asilar al filtrador fugitivo y, a la vez, dejaron fuera del foco de atención otras informaciones que sí interesan a la opinión pública venezolana por ser relevantes para su vida cotidiana.

Por su parte, el uso de adjetivos despectivos con respecto a una conducta, una institución o ideología hace entrever en el desarrollo mismo del discurso desinformativo que el emisor del mensaje está dotado de caracteres siempre positivos que constituyen una alternativa válida para enfrentarse a ese "mal" que primero se acuña y luego se denuncia. Sin embargo, es evidente que, aunque la posición del gobierno venezolano de proteger mediante asilo a Snowden, se apoya en el discurso oficial recurrente en contra de los Estados Unidos y en la ilegitimidad de la persecución internacional por espionaje, se produce una clara desinformación cuya intencionalidad es la de velar las discusiones de una ley diseñada, precisamente, para castigar las fugas de información.

También se evidencia claramente en la decisión de ofrecerle asilo a Snowden el ocultamiento de información en el caso, al plantearse en discusión legislativa una norma de carácter legal que sanciona la propia conducta defendida en el discurso oficial, aun cuando es en ese mismo discurso donde se omite la propuesta sancionatoria para el mismo acto que se avala.

\section{Evidencias de pragmática desinformativa}

El análisis cualitativo de base interpretativa del discurso mediático permitió desentrañar, bajo la perspectiva narrativa, diversas estratagemas de desinformación que 
ejecutaron los representantes de los tres países en análisis. Si bien la clasificación que se realizó en el marco conceptual del presente esfuerzo investigador es enunciativa, ha permitido la identificación del uso de al menos 11 estrategias léxico-semánticas y pragmalingüísticas, expresadas en la siguiente tabla:

Tabla 1: Técnicas de desinformación evidenciadas en el leak de Prism.

\begin{tabular}{|c|c|c|c|}
\hline Técnica & Estados Unidos & Alemania & Venezuela \\
\hline Distracción & & & $\mathrm{X}$ \\
\hline Omisión & & $\mathrm{X}$ & $\mathrm{X}$ \\
\hline Demonización & $\mathrm{X}$ & & $\mathrm{X}$ \\
\hline Sobresaturación & & & $\mathrm{X}$ \\
\hline Realidades Inventadas & & $\mathrm{X}$ & \\
\hline Adjetivos Disuasivos & & $\mathrm{X}$ & \\
\hline Eufemismos & & $\mathrm{X}$ & \\
\hline Mau Mauar & $\mathrm{X}$ & & \\
\hline Simplificación Conceptual & $\mathrm{X}$ & & \\
\hline Reforzamiento de la Autoculpabilidad & $\mathrm{X}$ & & \\
\hline Efecto Placebo & & $\mathrm{X}$ & \\
\hline
\end{tabular}

El gobierno de los Estados Unidos estaba concentrado en disuadir a otros países del otorgamiento de asilo a Snowden, bien para conseguir que el excolaborador regresara al país y pudiera ser puesto a disposición judicial, o para evitar que siguiera filtrando datos, fuera a los medios de comunicación o a otros gobiernos. Se pone en marcha la técnica del refuerzo de la culpabilidad acusándolo de revelación de secretos de Estado, incluso cuando éstos son constitutivos de atentado contra las propias libertades y soberanías extranjeras. Se desarrolla un proceso de demonización continua de Snowden al tildarlo de fugitivo de la justicia norteamericana y a una simplificación conceptual que evita el debate en profundidad sobre el acontecimiento, mientras se ejercen intensas presiones y amenazas a otros países al anunciarles las "graves consecuencias" que se derivarían de una posible concesión de asilo.

Por su parte, la canciller alemana Angela Merkel, reaccionaba a la información presentada por Snowden con indignación y rechazo inmediato contra los actos de espionaje que llevaba a cabo los Estados Unidos, aun cuando posteriormente Snowden desveló que el gobierno alemán había colaborado activamente con la NSA en el intercambio de información. Quedó demostrando por la fuerza de los hechos que la reacción inicial de la cancillería germana había sido estructurada con la función de desinformar a la opinión pública sobre la injerencia de los servicios secretos norteamericanos en la exposición de informaciones, sea por omisión o por mentir directamente sobre el desconocimiento en su reacción.

En otras latitudes, el gobierno venezolano reaccionaba a la información presentada por Snowden ofreciéndole el asilo inmediato, mientras su Parlamento discutía un proyecto de ley para imponer duras penas a cualquier persona que revelara secretos de Estado por cualquier medio. La intencionalidad del gobierno venezolano 
es evidente: no sólo le sirvía para mantener el discurso anti-imperialista que lo caracteriza desde 1999, sino también para desviar la atención de su opinión pública doméstica sobre los problemas económicos, sociales y políticos que aquejan al cotidiano devenir de la vida en el país.

\section{Conclusiones}

El actual ecosistema comunicativo alberga en su propio seno una carga desinformativa y de sobresaturación que hace ininteligible el trasfondo de todas las informaciones que se presentan en el podio discursivo social, creando un caldo de cultivo propicio para el desarrollo de desinformación (Aguaded y Romero-Rodríguez, 2015). Así, la desinformación pudiere ser una causa más que una consecuencia de la propia naturaleza y características del consumo informativo, por lo que es evidente que la audiencia objetivo de las informaciones pueden ser relativamente fáciles de engañar, sea por insuficiencia de análisis crítico, por la imposibilidad de acceder a las fuentes primarias de las informaciones o bien por la propia saturación e intoxicación en los canales comunicativos.

El caso Snowden podría ser sólo la punta del iceberg de todo un entramado de desinformación y vulneración de la privacidad de las telecomunicaciones, y deja en evidencia que el manejo actual del podio discursivo social intenta vehementemente modificar y alterar las percepciones de la opinión pública utilizando técnicas de desinformación y manipulación informativa. El normal decrecimiento del interés sobre los temas que realmente afectan a la sociedad viene dado a la vez por el advenimiento de una lógica de incompletitud de las informaciones, aunado a la propia dinámica social, por lo que la pérdida de vigencia de dicha información viene acompañada por una sobresaturación de otras informaciones -importantes o no- en nuestro acontecer diario, circunstancia que funciona como un óptimo caldo de cultivo para que quienes tienen acceso al podio discursivo mediático alteren la agenda setting y por ende la propia construcción de las realidades.

\section{Referencias bibliográficas}

AGUADED, Ignacio; ROMERO-RODRÍGUEZ, Luis M (2015). Mediamorfosis y desinformación en la infoesfera: Alfabetización mediática, digital e informacional ante los cambios de hábitos de consumo informativo. En: Education in the Knowledge Society (EKS), 16 (1), p.44-57. (doi: http://doi.org/4ht).

ALLAN, Keith; BURRIDGE, Kate (1991). Euphemism and dysphemism: language used as shield and weapon. Oxford: Oxford University Press.

BELLOVIN, Steven M. et al. (2008). Risking Communications Security: Potential Hazards of the Protect America Act. En: Institute of Electrical and Electronics Engineers Journal of Security and Privacy, 6 (1), p. 24-32.

BERGER, Peter .L; LUCKMANN, Thomas (2003). La construcción social de la realidad. Buenos Aires: Amorrortu. 
BRAIKER, Harriet (2003). Who's Pulling your Strings? How to Break the Cycle of Manipulation. New York: McGraw Hill.

DURANDÍN, Guy (1995). La información, la desinformación y la realidad. Madrid: Paidós Ibérica.

ELLIS, Carollyn; BOCHNER, Arthur P (2006). Analyzing analytic autoetnography: An autopsy. En: Journal of Contemporary Ethnography, 35 (4), p.429-449.

GARCÍA-AVILÉS, José A (2009). La Desinformación. En: HERRERO, Julio C. (ed.) Manual de Teoría de la Información y la Comunicación. Madrid: Universitas, pp. 332-335.

GILLESPIE, Tarleton (2010). The politics of 'platforms'. En: New Media \& Society, 12 (3), p. 347-364. (doi: http://doi.org/b4sxdq).

GILOVICH, Thomas (1993). How We Know Whats Isn't So. New York: The Free Press.

GREENE, Michael (2014). Where Has Privacy Gone? How Surveillance Programs Threaten Expectations Of Privacy. En: The John Marshall Journal of Information Technology \& Privacy Law, 30(4), p.795-826.

GRIJELMO, Alex (2000). La seducción de las palabras. Madrid: Taurus.

HINDMAN, Matthew (2013). Personalization and the future of news. Working Paper. Robert Schuman Center, European University Institute, Florence. [http://goo. gl/8plL4S] (07/11/2015).

KARPF, David; et al (2015). The Role of Qualitative Methods in Political Communication Research: Past, Present, and Future. En: International Journal of Communication, v. 9, p. 1888-1906.

LEE, Karen V (2006). A fugue about grief. En: Qualitative Inquiry, 12 (6), p. 66-80. (doi: http://doi.org/b9xj74)

MORAL-SANTAELLA, Cristina (2015). Estrategias para resistir a la crisis de confianza en la investigación cualitativa actual. Educación XXI, 19 (1), 1-24. (DOI: http://doi.org/84t)

PREIBUSCH, Sören (2015). Privacy behaviors after Snowden. Communications of the ACM, 58(5), 48-55. (DOI: http://doi.org/835)

RICHARDSON, Laurel; ADAMS-ST.PIERRE, Elizabeth (2000). Writing: A method of inquiry. En: DENZIN, Norman K; LINCOLN, Yvonna S (eds.), Handbook of Qualitative Research, p. 923-943. London: Sage.

ROMERO-RODRÍGUEZ, Luis M (2013). Hacia un estado de la cuestión de las investigaciones sobre desinformación / misinformación. Correspondencias \& Análisis, 3, p. 319-342.

ROMERO-RODRÍGUEZ, Luis M (2015). Pragmática de la desinformación: Estratagemas e incidencia de la calidad informativa de los medios. Tesis Doctoral. Repositorio Arias Montano, Universidad de Huelva.

ROMERO-RODRÍGUEZ, Luis M; DE CASAS, Patricia; TORRES-TOUKOUMIDIS, Ángel (2015). Estereotipos, tópicos y lenguaje de la programación sensacionalista en la televisión: Programa «Corazón» de TVE. Alteridad, 10,1, 31-43. (DOI: http://doi.org/84f).

SAINI, Michael; SHLONSKY, Aron (2012). Systematic synthesis of qualitative research. New York: Oxford University Press.

SEARLE, John (1997). La construcción de la realidad social. Barcelona: Paidós. 
STEMPEL, Guido (1989). Content Analysis. En: WESTLEY J.W; STEMPEL, Guido, Research Methods in Mass Communications, p. 240-248. New Jersey: Prentice-Hall.

TUROW, Joseph (2011). The daily you: How the new advertising industry is defining your identity and your world. New Haven, CT: Yale University Press.

WATZLAWICK, Paul (2003). ¿Es real la realidad? Barcelona: Herder.

WOLFE, Tom (2011). La Izquierda Exquisita \& Maumauando al Parachoques. Madrid: Anagrama. 